\title{
CLUSTER AUTOMORPHISMS AND COMPATIBILITY OF CLUSTER VARIABLES
}

\author{
IBRAHIM ASSEM AND VASILISA SHRAMCHENKO \\ Département de mathématiques, Université de Sherbrooke, Sherbrooke, Qc, J1K 2R1, Canada \\ e-mails: ibrahim.assem@usherbrooke.ca; vasilisa.shramchenko@usherbrooke.ca \\ and RALF SCHIFFLER \\ Department of Mathematics, University of Connecticut, Storrs, CT 06269-3009, USA \\ e-mail: schiffler@math.uconn.edu \\ Dedicated to the memory of Andrei Zelevinsky
}

(Received 17 July 2013; accepted 4 November 2013)

\begin{abstract}
In this paper, we introduce a notion of unistructural cluster algebras, for which the set of cluster variables uniquely determines the clusters, as well as the notion of weak unistructural cluster algebras, for which the set of cluster variables determines the clusters, provided that the type of the cluster algebra is fixed. We prove that, for cluster algebras of the Dynkin type, the two notions of unistructural and weakly unistructural coincide, and that cluster algebras of rank 2 are always unistructural. We then prove that a cluster algebra $\mathcal{A}$ is weakly unistructural if and only if any automorphism of the ambient field, which restricts to a permutation of cluster variables of $\mathcal{A}$, is a cluster automorphism. We also investigate the FominZelevinsky conjecture that two cluster variables are compatible if and only if one does not appear in the denominator of the Laurent expansions of the other.
\end{abstract}

2010 Math Classification Numbers 13F60, 16G20.

1. Introduction. Fomin and Zelevinsky [12] introduced cluster algebras in 2002 which since then have been proved to be related to various areas of mathematics such as Combinatorics, Representation Theory of Algebras, Mathematical Physics, Teichmüller Theory and many others.

In [4], Assem et al. introduced the notion of cluster automorphism. Let $\mathcal{A}$ be a cluster algebra. A cluster automorphism of $\mathcal{A}$ is a $\mathbb{Z}$-automorphism of the algebra $\mathcal{A}$ mapping a cluster to a cluster and commuting with mutations. In [19], Saleh defines another notion of automorphism of a cluster algebra: This is an automorphism of the ambient field which restricts to a permutation of the set of cluster variables.

In the present paper, we investigate the following conjecture.

Conjecture 1.1. Let $\mathcal{A}$ be a cluster algebra. Then $f: \mathcal{A} \rightarrow \mathcal{A}$ is a cluster automorphism if and only if $f$ is an automorphism of the ambient field which restricts to a permutation of the set of cluster variables.

One implication of this conjecture follows from the fact that cluster automorphisms map each cluster to a cluster [5, Corollary 2.7], so in particular cluster automorphisms permute the cluster variables. 
The other implication led us to consider the following more general question. We say that a cluster algebra is unistructural if the set of cluster variables determines the cluster algebra structure, that is, there exists a unique decomposition of the set of cluster variables into clusters. The following conjecture is natural.

Conjecture 1.2. Any cluster algebra is unistructural.

We also define a notion of weak unistructurality: A cluster algebra $\mathcal{A}$ is weakly unistructural if its set of cluster variables does not coincide with the set of cluster variables of a cluster algebra $\mathcal{A}^{\prime}$ of the same type as $\mathcal{A}$, that is, with $\mathcal{A}$ and $\mathcal{A}^{\prime}$, both containing seeds with the same quiver. Clearly, a unistructural cluster algebra is weakly unistructural, so the statement that every cluster algebra is weakly unistructural would follow from Conjecture 1.2.

CONJECTURE 1.3. The following three conditions are equivalent for a cluster algebra $\mathcal{A}$ :

(1) $\mathcal{A}$ is weakly unistructural;

(2) $\mathcal{A}$ is unistructural;

(3) if $f: \mathcal{A} \rightarrow \mathcal{A}$ is an automorphism of the ambient field which restricts to a permutation of the set of cluster variables of $\mathcal{A}$, then $f$ is a cluster automorphism.

We show that this conjecture holds for cluster algebras of Dynkin type. We also show the equivalence of items (1) and (3) of Conjecture 1.3 in general, namely we prove the following theorem.

THEOREM 1.4. A cluster algebra $\mathcal{A}$ is weakly unistructural if and only if any automorphism of the ambient field which restricts to a permutation of cluster variables of $\mathcal{A}$ is a cluster automorphism.

In particular, we prove that cluster algebras of rank 2 are unistructural and so in this case, a permutation of cluster variables induces a cluster automorphism.

Since the unistructurality Conjecture 1.2 deals with arranging cluster variables into clusters, this led us to consider a related problem of compatibility of cluster variables. Recall that two cluster variables are called compatible if there exists a cluster containing both of them. Here we investigate Fomin and Zelevinsky's Conjecture 7.4(2) [13], which we restate as follows.

Conjecture 1.5. Let $\mathcal{A}$ be a cluster algebra and $x, x^{\prime}$ be two cluster variables. Then $x$ and $x^{\prime}$ are compatible if and only if, for any cluster $\mathbf{x}$ containing $x$, the expansion $\mathcal{L}\left(x^{\prime}, \mathbf{x}\right)$ of $x^{\prime}$ as Laurent polynomial in $\mathbf{x}$ (in reduced form) is of the form $\frac{P(\mathbf{x})}{m(\mathbf{x} \backslash\{x\})}$, where $P$ is a polynomial in the variables of $\mathbf{x}$ and $m$ is a monomial in the variables of $\mathbf{x}$ excluding $x$.

In the sequel, we shall simply say that $\mathcal{L}\left(x^{\prime}, \mathbf{x}\right)$ has no $x$ in the denominator to express that $\mathcal{L}\left(x^{\prime}, \mathbf{x}\right)$ is of the previous form.

This Conjecture 1.5 is proved for the case of cluster algebras arising from surfaces, see [11, Theorem 8.6].

We prove the following theorem.

THEOREM 1.6. Let $\mathcal{A}_{Q}$ be an acyclic cluster algebra and $x, x^{\prime}$ are two cluster variables.

(a) If $x$ is transjective, then $x$ and $x^{\prime}$ are compatible if and only if for any cluster $\mathbf{x}$ containing $x$, the Laurent expansion $\mathcal{L}\left(x^{\prime}, \mathbf{x}\right)$ has no $x$ in its denominator. 
(b) If $x$ is regular and $Q$ is Euclidean, then $x$ and $x^{\prime}$ are compatible if and only if, for any cluster $\mathbf{x}^{\prime}$ containing $x^{\prime}$, the Laurent expansion $\mathcal{L}\left(x, \mathbf{x}^{\prime}\right)$ has no $x^{\prime}$ in its denominator.

Observe that the statement of Theorem 1.6 is not symmetric in $x$ and $x^{\prime}$. This led us to the following conjecture which is weaker than Conjecture 1.5.

Conjecture 1.7. Let $\mathcal{A}$ be a cluster algebra and $x, x^{\prime}$ be two cluster variables. Assume that for any cluster $\mathbf{x}$ containing $x$, the Laurent expansion $\mathcal{L}\left(x^{\prime}, \mathbf{x}\right)$ has no $x$ in its denominator. Then for any cluster $\mathbf{x}^{\prime}$ containing $x^{\prime}$, the Laurent expansion $\mathcal{L}\left(x, \mathbf{x}^{\prime}\right)$ has no $x^{\prime}$ in its denominator.

The paper is organised as follows. In Section 2, we recall some preliminary notions and facts that will be useful for the proof of our results. Section 3 is devoted to part $(a)$ of Theorem 1.6, while part $(b)$ is proven in Section 4. Finally, we discuss unistructurality and automorphisms in Section 5.

\section{Preliminaries.}

2.1. Cluster algebras. Let $Q$ be a finite connected quiver without loops or twocycles. We denote by $n=\left|Q_{0}\right|$ the number of points of $Q$, the points are denoted by $1,2, \ldots, n$. Let $\mathbf{x}=\left\{x_{1}, \ldots, x_{n}\right\}$ be a set of $n$ variables, called a cluster, where we agree that the variable $x_{i}$ corresponds to the point $i$ of the quiver. The pair $(\mathbf{x}, Q)$ is called a seed. The field $\mathcal{F}=\mathbb{Q}\left(x_{1}, \ldots, x_{n}\right)$ of rational functions in $x_{1}, \ldots, x_{n}$ is called the ambient field.

Let $k$ be such that $1 \leq k \leq n$. The seed mutation $\mu_{k}$ in direction $k$ transforms $(\mathbf{x}, Q)$ into $\mu_{k}(\mathbf{x}, Q)=\left(\mathbf{x}^{\prime}, Q^{\prime}\right)$ defined as follows:

First, $Q^{\prime}$ is obtained from $Q$ by applying the following operations:

- for any path $i \rightarrow k \rightarrow j$, a new arrow $i \rightarrow j$ is inserted;

- all arrows incident to $k$ are reversed;

- all ensuing two cycles are deleted. relation

Next, $\mathbf{x}^{\prime}=\left(\mathbf{x} \backslash\left\{x_{k}\right\}\right) \bigcup\left\{x_{k}^{\prime}\right\}$, where $x_{k}^{\prime} \in \mathcal{F}$ is defined by the so-called exchange

$$
x_{k}^{\prime}=\frac{\prod_{i \rightarrow k} x_{i}+\prod_{k \rightarrow i} x_{i}}{x_{k}},
$$

where products are taken over arrows entering and leaving $k$ respectively.

Iterating this procedure, we obtain a set $\left\{\left(\mathbf{x}_{\alpha}, Q_{\alpha}\right)\right\}_{\alpha}$ of seeds, where the $\mathbf{x}_{\alpha}$ are the clusters and the $Q_{\alpha}$ are the exchange quivers. The cluster algebra $\mathcal{A}=\mathcal{A}(\mathbf{x}, Q)$, with initial seed $(\mathbf{x}, Q)$ is the $\mathbb{Z}$-subalgebra of $\mathcal{F}$ generated by the union $\mathcal{X}=\bigcup_{\alpha} \mathbf{x}_{\alpha}$ of all possible clusters obtained from $\mathbf{x}$ by successive mutations. Elements of $\mathcal{X}$ are called cluster variables. Two cluster variables are called compatible if there exists a cluster containing both.

One of the most remarkable results of the theory is the Laurent phenomenon [12] which asserts that for any cluster algebra $\mathcal{A}$ and any seed $(\mathbf{x}, Q)$ of $\mathcal{A}$, each cluster variable $x$ of $\mathcal{A}$ is a Laurent polynomial over $\mathbb{Z}$ in the cluster variables from 
$\mathbf{x}=\left(x_{1}, \ldots, x_{n}\right)$, that is, $x$ can be written as

$$
x=\mathcal{L}(x, \mathbf{x})=\frac{p(\mathbf{x})}{x_{1}^{d_{1}}, \ldots, x_{n}^{d_{n}}}
$$

as a reduced fraction, that is, $p$ is not divisible by any of the $x_{i}$. The vector $\mathbf{d}=$ $\left(d_{1}, \ldots, d_{n}\right)$ is called the denominator vector of $x$, denoted by $\operatorname{den}(x)$.

A famous conjecture is the so-called positivity conjecture. It says that, for any cluster algebra $\mathcal{A}$, any cluster variable $x$ and any seed $(\mathbf{x}, Q)$ of $\mathcal{A}$, the numerator $p(\mathbf{x})$ of $\mathcal{L}(x, \mathbf{x})$ has coefficients which are non-negative integers. The positivity conjecture has been proved in several important cases, most notably for cluster algebras arising from surfaces [17] and for acyclic cluster algebras [14], that is, cluster algebras arising from a quiver which can be transformed to an acyclic quiver using a sequence of mutations, and finally in [15] for all cluster algebras arising from quivers.

2.2. The cluster category. Let $Q$ be an acyclic quiver, and $k$ be an algebraically closed field. We denote by $k Q$ the path algebra of $Q$, by $\bmod k Q$ the category of finitely generated $k Q$-modules and by $\mathcal{D}=\mathcal{D}^{b}(\bmod k Q)$ the bounded derived category over $\bmod k Q$. The cluster category $\mathcal{C}=\mathcal{C}_{Q}$ is the orbit category of $\mathcal{D}$ under the action of the automorphism $\tau^{-1}[1]$, where $\tau$ is the Auslander-Reiten translation and [1] is the shift in $\mathcal{D}$, see [7]. Then $\mathcal{C}$ is a triangulated 2-Calabi-Yau category having almost split triangles. The Auslander-Reiten quiver $\Gamma(\mathcal{C})$ of $\mathcal{C}$ is the quotient of $\Gamma(\mathcal{D})$ under the action of the quiver automorphism induced by the functor $\tau^{-1}[1]$. Note that the indecomposable objects of $\mathcal{C}$ may be identified with indecomposable $k Q$-modules or with indecomposable summands of $k Q[1]=\left\{P_{i}[1] \mid i \in Q_{0}\right\}$, the shifts of the indecomposable projective $k Q$-modules. Then $\Gamma(\mathcal{C})$ always has a unique component containing all objects of $k Q[1]$. This component is called transjective. If $Q$ is Dynkin, then $\Gamma(\mathcal{C})$ reduces to the transjective component (and is finite). Otherwise the transjective component is of the form $\mathbb{Z} Q$ (see [5, VIII 1.1]) and there are infinitely many other components called regular, which are either stable tubes (if $Q$ is Euclidean) or of type $\mathbb{Z} \mathbb{A}_{\infty}$ (if $Q$ is wild).

Let $n=\left|Q_{0}\right|$. A (basic) tilting object $T$ in $\mathcal{C}_{Q}$ is an object of the form $T=\oplus_{i=1}^{n} T_{i}$, where $T_{i}$ are indecomposable non-isomorphic objects of $\mathcal{C}$ such that $\operatorname{Hom}_{\mathcal{C}}\left(T_{i}, T_{j}[1]\right)=$ 0 for all $i, j$. In particular, we have $\operatorname{Hom}_{\mathcal{C}}\left(T_{i}, T_{i}[1]\right)=0$ for all $i$, that is, each $T_{i}$ is a rigid object of $\mathcal{C}$. With each tilting object $T$ is associated a map

$$
X_{?}^{T}: \mathcal{C}_{0} \rightarrow \mathbb{Z}\left[x_{1}^{ \pm 1}, \ldots, x_{n}^{ \pm 1}\right]
$$

called the cluster character. This map induces a bijection between the indecomposable rigid objects of $\mathcal{C}$ and the cluster variables in the cluster algebra $\mathcal{A}(\mathbf{x}, Q)$. This bijection also induces a bijection between the tilting objects in $\mathcal{C}$ and the clusters of $\mathcal{A}(\mathbf{x}, Q)$ (see $[10,18])$.

An algebra $\mathcal{B}$ is called cluster-tilted of type $Q$ if there exists a tilting object $T$ in $\mathcal{C}_{Q}$ such that $\mathcal{B}=\operatorname{End}_{\mathcal{C}_{Q}} T$. Let $(\operatorname{add}(\tau T))$ be the ideal of $\mathcal{C}=\mathcal{C}_{Q}$ consisting of those morphisms factoring through objects of $\operatorname{add}(\tau T)$. Then the functor $\operatorname{Hom}_{\mathcal{C}}(T,-)$ induces an equivalence $\mathcal{C} /(\operatorname{add}(\tau T)) \simeq \bmod B\left(\right.$ see [9]). For any $i \in Q_{0}$, we denote by $S_{i}$ the simple $B$-module corresponding to $i$. We denote by $\langle-,-\rangle$ the bilinear form on 
$\bmod B$ defined by

$$
\langle M, N\rangle=\operatorname{dim} \operatorname{Hom}_{B}(M, N)-\operatorname{dim} \operatorname{Ext}_{B}^{1}(M, N)
$$

for any $B$-modules $M$ and $N$. Further, we write

$$
\langle M, N\rangle_{a}=\langle M, N\rangle-\langle N, M\rangle,
$$

that is, $\langle-,-\rangle_{a}$ is the anti-symmetrised form of $\langle-,-\rangle$. Let $\mathbf{e} \in \mathbb{N} Q_{0}$ and $\operatorname{Gr}_{\mathbf{e}} M$ denote the set of submodules of $M$ having dimension-vector equal to e. This set is a projective variety, called the Grassmannian of submodules of $M$ of dimension vector e. We denote by $\chi\left(\mathrm{Gr}_{\mathbf{e}} M\right)$ its Euler-Poincaré characteristic (with respect to the singular cohomology if $k$ is the field of complex numbers, and to the étale cohomology if $k$ is arbitrary). Then the cluster character $X_{?}^{T}$ is the unique map such that:

(a) $X_{T_{i}[1]}^{T}=x_{i}$ for any $i \in Q_{0}$;

(b) if $M$ is indecomposable and not isomorphic to any $T_{i}[1]$, then

$$
X_{M}^{T}=\sum_{e \in \mathbb{N} Q_{0}} \chi\left(\operatorname{Gr}_{e}\left(\operatorname{Hom}_{\mathcal{C}}(\mathrm{T}, \mathrm{M})\right)\right) \prod_{i \in Q_{0}} x_{i}^{\left\langle S_{i}, e\right\rangle_{a}-\left\langle S_{i}, \operatorname{Hom}_{\mathcal{C}}(T, M)\right\rangle} ;
$$

(c) for any two objects $M, N$ in $\mathcal{C}$,

$$
X_{M \oplus N}^{T}=X_{M}^{T} X_{N}^{T}
$$

A cluster variable $x$ in $\mathcal{A}(\mathbf{x}, Q)$ is called transjective (or regular) if $x=X_{M}^{T}$ with $M$ an indecomposable rigid transjective (or regular respectively) object of $\mathcal{C}$.

It is shown in [8] that if $T$ has only transjective direct summands and $x=X_{M}^{T}$ is any cluster variable, then

$$
\operatorname{den}(x)=\operatorname{dim} \operatorname{Hom}_{\mathcal{C}}(T, M)
$$

Moreover, if $Q$ is Euclidean, and no indecomposable regular direct summand of $T$ is of quasi-length $r-1$ lying in a tube of rank $r$, then this equality holds for all cluster variables.

\section{The transjective case.}

3.1. The motivating example and Conjecture 1.5. In [3], it was asked when indecomposable rigid modules over cluster-tilted algebras are uniquely determined by their composition factors. This is related to problem 7.6 of [13]. The main result of [3] says that if $B$ is a cluster-tilted algebra and $M, N$ are indecomposable transjective modules (in particular, are rigid), then $M \cong N$ if and only if $\operatorname{dim} M=\operatorname{dim} N$. This is not true if $M, N$ are not transjective. Indeed, let $B$ be the cluster-tilted algebra of type $\tilde{\mathbb{A}}_{1,2}$ given by the quiver

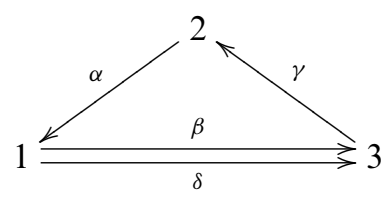


bound by $\alpha \beta=\beta \gamma=\gamma \alpha=0$. Then $\Gamma(\bmod B)$ contains a tube of rank 2 containing the projective-injective module $P_{2}=I_{2}$ corresponding to point 2 of the quiver:

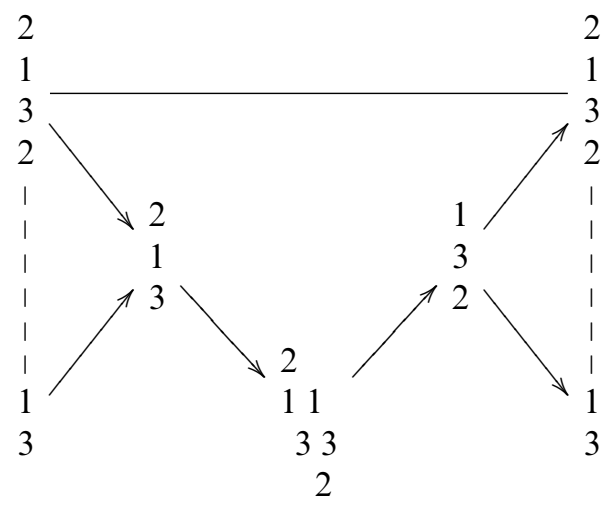

where we identify along the dotted lines and $\begin{aligned} & 2 \\ & 1 \\ & 3 \\ & 2\end{aligned}$ lies on the mouth. Then $\begin{array}{cc}2 & 1 \\ 3 & \text { and } \\ 3 & 2\end{array}$ are non-isomorphic indecomposable rigid $B$-modules with the same dimension vector. Let $T_{2}$ be the object in the cluster category corresponding to the projective $B$-module $P_{2}$, then the corresponding cluster variable is

$$
\frac{2 x_{1} x_{3}+x_{3}^{2}+x_{2}+x_{1}^{2}}{x_{1} x_{2} x_{3}}
$$

In particular, its denominator vector is $(1,1,1)$, while its dimension vector is $(1,2,1)$. Note, however, that in this example the $i$ th component of the denominator vector is non-zero and also the $i$ th component of the dimension vector is non-zero. This led us to consider conjecture 7.4(2) of [13], which we have restated as Conjecture 1.5, see Introduction.

One implication of the conjecture is easy.

LEMMA 3.1. Let $\mathcal{A}$ be a cluster algebra and $x, x^{\prime}$ be two cluster variables. If $x, x^{\prime}$ are compatible then, for any cluster $\mathbf{x}$ containing $x, \mathcal{L}\left(x^{\prime}, \mathbf{x}\right)$ has no $x$ in the denominator.

Proof. Let $\mathbf{x}$ be a cluster containing $x$. Because $x$ and $x^{\prime}$ are compatible, there exists a sequence of mutations (not involving $\mu_{x}$ ) such that $\mathbf{x}$ is mutated to a new cluster $\mathbf{x}^{\prime}$ containing $x$ and $x^{\prime}$ simultaneously. Expanding $x^{\prime}$ in terms of the original cluster $\mathbf{x}$, we get $\mathcal{L}\left(x^{\prime}, \mathbf{x}\right)$ of the required form.

3.2. Proof of Theorem 1.6(a). We start with an easy technical lemma. Throughout, we assume that $\mathcal{A}=\mathcal{A}(\mathbf{x}, Q)$ is an acyclic cluster algebra and denote by $\mathcal{C}=\mathcal{C}_{Q}$ the associated cluster category.

Lemma 3.2. Let $x, x^{\prime}$ be two cluster variables in $\mathcal{A}$, and $T$ be a tilting object in $\mathcal{C}$ such that $X_{T_{i}[1]}^{T}=x$. If $M^{\prime} \in \mathcal{C}$ is such that $X_{M^{\prime}}^{T}=x^{\prime}$, then $x$ and $x^{\prime}$ are compatible if and only if $\operatorname{Hom}_{\mathcal{C}}\left(T_{i}, M^{\prime}\right)=0$. 
Proof. Indeed, $x$ and $x^{\prime}$ are compatible if and only if the corresponding objects $T_{i}[1]$ and $M^{\prime}$ are compatible, that is, $\operatorname{Hom}_{\mathcal{C}}\left(T_{i}[1], M^{\prime}[1]\right)=0$. But this is equivalent to $\operatorname{Hom}_{\mathcal{C}}\left(T_{i}, M^{\prime}\right)=0$.

We are now able to state and prove the following theorem.

THEOREM 3.3. Let $\mathcal{A}$ be a cluster algebra, and $x, x^{\prime}$ be two cluster variables with $x$ transjective. Then $x$ and $x^{\prime}$ are compatible if and only if, for any cluster $\mathbf{x}$ containing $x$, the Laurent expansion $\mathcal{L}\left(x^{\prime}, \mathbf{x}\right)$ has no $x$ in the denominator.

Proof. Because of Lemma 3.1, we only need to prove the sufficiency. Assume to the contrary that $x, x^{\prime}$ are not compatible, and let $\mathbf{x}$ be a transjective cluster containing $x$. Such a cluster always exists, take for instance a slice in the cluster category containing the object corresponding to $x$, see [1]. Let $T \in \mathcal{C}$ be the tilting object corresponding to $\mathbf{x}$. Then $T$ is transjective and there exists an indecomposable summand $T_{i}$ of $T$ such that $X_{T_{i}[1]}^{T}=x$. Let also $M^{\prime}$ be the object in $\mathcal{C}$ such that $X_{M^{\prime}}^{T}=x^{\prime}$. Then, because of [8] (see Section 2 above), we have $\operatorname{den}\left(x^{\prime}\right)=\operatorname{dim} \operatorname{Hom}_{\mathcal{C}}\left(T, M^{\prime}\right)$. On the other hand, because of the previous lemma, the incompatibility of $x$ and $x^{\prime}$ implies that $\operatorname{Hom}_{\mathcal{C}}\left(T_{i}, M^{\prime}\right) \neq 0$. This shows that

$$
\mathcal{L}\left(x^{\prime}, \mathbf{x}\right)=\frac{P(\mathbf{x})}{x^{d} m(\mathbf{x} \backslash\{x\})},
$$

with $d>0$. The proof is now complete.

The main application of this theorem is to cluster automorphisms (see Section 5). For the time being, we obtain an obvious corollary asserting the truth of the previous conjecture in two particular cases.

COROLlary 3.4. Let $\mathcal{A}$ be a cluster algebra of the Dynkin type, or of rank 2, then two cluster variables $x$ and $x^{\prime}$ are compatible if and only if, for any cluster $\mathbf{x}$ containing $x$, the Laurent expansion $\mathcal{L}\left(x^{\prime}, \mathbf{x}\right)$ has no $x$ in the denominator.

Proof. Indeed, in these cases, all cluster variables are transjective.

3.3. Remark. The statement of the theorem above is clearly not symmetric: It says that if one of the variables is transjective, then compatibility means that the Laurent expansion of the other does not have the first in the denominator.

\section{The tame case.}

4.1. Preliminaries on the cluster category. As in Section 3 , we let $\mathcal{A}$ be a cluster algebra of type $Q$, and we denote by $\mathcal{C}$ the associated cluster category. Throughout this section, we assume that $Q$ is a Euclidean quiver.

Assume that $T_{1}$ is an indecomposable rigid object of quasi-length $r-1$ lying in a tube $\mathcal{T}$ of $\Gamma(\mathcal{C})$ of rank $r$. We denote by $T_{1} \longrightarrow T_{2} \longrightarrow \cdots \longrightarrow T_{r-1}$ the sectional path from $T_{1}$ to the mouth of $\mathcal{T}$ and assume that $T$ is a tilting object having all the $T_{i}$ (with $1 \leq i \leq r-1$ ) as summands. Such an object exists because the $T_{i}$ are clearly compatible in $\mathcal{C}$.

Consider the set $\Delta$ of all indecomposables in $\mathcal{T}$ lying on a path of irreducible morphisms from $T_{1}$ to $\tau^{2} T_{1}$ of length equal to $2 r-4$. Observe that, for any 
$M \in \Delta$, we have $\operatorname{Hom}_{\mathcal{C}}\left(M, \tau^{2} T_{1}\right) \neq 0$. Actually, if $M$ is a rigid object in $\mathcal{T}$ such that $\operatorname{Hom}_{\mathcal{C}}\left(T_{1}, M\right) \neq 0$, then $M$ belongs to $\Delta$ (and even to the 'upper half' of $\Delta$ ). Note that in [6], the authors define the notion of a wing $\mathcal{W}_{\tau T_{1}}$ attached to $T_{1}$. This is related to $\Delta$ as follows: If $X$ is a rigid object in $\mathcal{T}$, then $X \in \Delta$ if and only if $X \notin \mathcal{W}_{\tau T_{1}}$.

LEMMA 4.1. $\Delta$ contains no direct summand of $\tau T$.

Proof. Indeed, if $\tau T_{i} \in \Delta$ for some $i$ such that $1 \leq i<r$, then there is a morphism from $T_{1}$ to $\tau T_{i}$, and thus $\operatorname{Ext}_{\mathcal{C}}^{1}\left(T_{1}, T_{i}\right) \neq 0$, a contradiction.

Let $U_{1}$ denote the unique direct predecessor of $T_{1}$ of quasi-length $r$. Then we have the following picture.

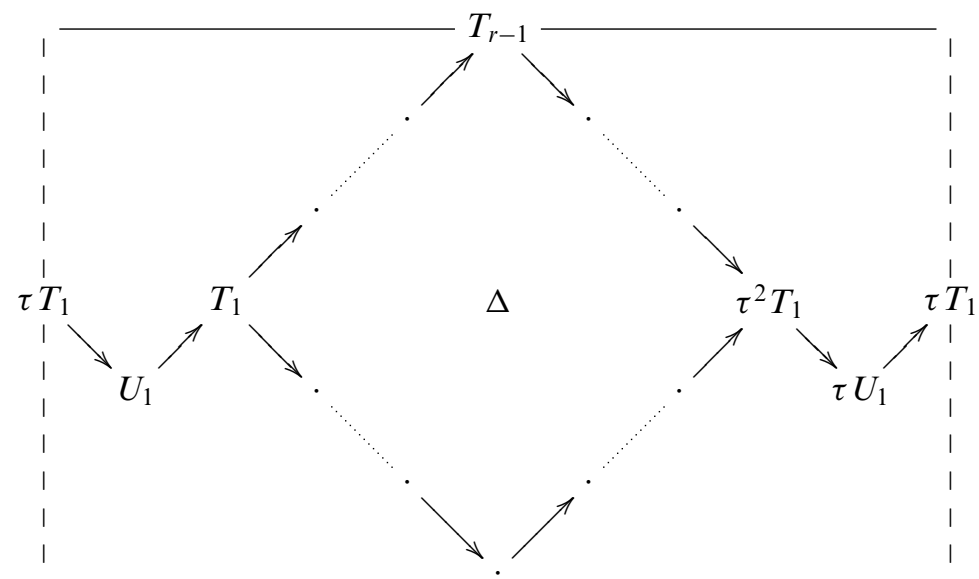

where we identify along the vertical dashed lines and $T_{r-1}$ lies on the mouth of $\mathcal{T}$.

LEMMA 4.2. With the notation above, we have

(a) $\operatorname{dim} \operatorname{Hom}_{\mathcal{C}}\left(T_{1}, M\right)=2$ for every $M \in \Delta$.

(b) $\operatorname{dim} \operatorname{Hom}_{\mathcal{C}}\left(U_{1}, M\right)=2$ if $M \in \Delta$ does not lie on the sectional path from the mouth to $\tau U_{1}$.

(c) $\operatorname{dim} \operatorname{Hom}_{\mathcal{C}}\left(U_{1}, M\right)=1$ if $M \in \Delta$ lies on the sectional path from the mouth to $\tau U_{1}$.

Proof. Recall that, for all $X, Y \in \mathcal{T}$,

$$
\begin{aligned}
\operatorname{Hom}_{\mathcal{C}}(X, Y) & =\operatorname{Hom}_{\mathcal{D}}(X, Y) \oplus \operatorname{Hom}_{\mathcal{D}}(\tau X[-1], Y) \\
& =\operatorname{Hom}_{k Q}(X, Y) \oplus \operatorname{Ext}_{k Q}^{1}(\tau X, Y) .
\end{aligned}
$$

Now, because $\mathcal{T}$ is standard in $\bmod k Q$, for $M \in \Delta$, we have

$$
\operatorname{dim} \operatorname{Hom}_{k Q}\left(T_{1}, M\right)=1,
$$

while $\operatorname{dim} \operatorname{Ext}_{k Q}^{1}\left(\tau T_{1}, M\right)=\operatorname{dim} \operatorname{Hom}_{k Q}\left(M, \tau^{2} T_{1}\right)=1$, where we have applied the Auslander-Reiten formula. Similarly, for $M \in \Delta$, we have

$$
\operatorname{dim} \operatorname{Hom}_{k Q}\left(U_{1}, M\right)=1,
$$


while $\operatorname{dim} \operatorname{Ext}_{k Q}^{1}\left(\tau U_{1}, M\right)=\operatorname{dim} \operatorname{Hom}_{k Q}\left(M, \tau^{2} U_{1}\right)$ is equal to 1 or 0 according to the cases considered in (b) or (c) respectively. Here we use essentially that $T_{1}$ and $U_{1}$ are of quasi-length $r-1$ and $r$ respectively.

4.2. Passing to cluster-tilted algebras. Now, let $B=\operatorname{End}_{\mathcal{C}} T$ be the cluster-tilted algebra corresponding to the tilting object $T$ in $\mathcal{C}$ considered in Section 4.1. Then $P_{1}=\operatorname{Hom}_{\mathcal{C}}\left(T, T_{1}\right)$ is projective, and there is a sectional path of projective modules of length $r-1$ from $P_{1}$ to the mouth of the tube. On the other hand, $I_{1}=\operatorname{Hom}_{\mathcal{C}}\left(T, \tau^{2} T_{1}\right)$ is the injective module corresponding to $P_{1}$ (see [2, Lemma 5]).

Let $\Omega=\left\{\operatorname{Hom}_{\mathcal{C}}(T, M) \mid M \in \Delta\right\}$. Observe that, because of Lemma 4.1, for every $M \in \Delta$, the $B$-module $\operatorname{Hom}_{\mathcal{C}}(T, M)$ is indecomposable. Let $R_{1}=\operatorname{Hom}_{\mathcal{C}}\left(T, U_{1}\right)$. Then $R_{1}$ is equal to the radical of the indecomposable projective $B$-module $P_{1}$ because the unique direct successor $P_{2}=\operatorname{Hom}_{\mathcal{C}}\left(T, T_{2}\right)$ of $P_{1}$ of smaller quasi-length is projective.

LEMMA 4.3. With the above notation, for every $N \in \Omega$, we have

(a) $\operatorname{dim} \operatorname{Hom}_{B}\left(P_{1}, N\right)=2$.

(b) $\operatorname{dim} \operatorname{Hom}_{B}\left(R_{1}, N\right)=1$.

Proof. We recall from Section 2 that $\bmod B \cong \mathcal{C} / \operatorname{add} \tau T$. Let $M \in \mathcal{C}$ be such that $\operatorname{Hom}_{\mathcal{C}}(T, M)=N$. We first observe that since $T$ is a tilting object in $\mathcal{C}$, no morphism from $T_{1}$ to $M$ factors through add $\tau T$. Therefore, Lemma 4.1 implies (a).

In order to prove (b), we recall that, according to Lemma 4.1, a basis of the vector space $\operatorname{Hom}_{\mathcal{C}}\left(U_{1}, M\right)$ consists of at most two morphisms, one of them constituting a basis of $\operatorname{Hom}_{k Q}\left(U_{1}, M\right)$ and the other (if non-zero) constituting a basis of $\operatorname{Ext}_{k Q}^{1}\left(\tau U_{1}, M\right)$. Now note that if $g$ is a basis vector in $\operatorname{Hom}_{k Q}\left(U_{1}, M\right)$, then it clearly does not factor through add $\tau T$ and so $\operatorname{Hom}_{\mathcal{C}}(T, g): R_{1} \rightarrow N$ is non-zero. Thus, in order to prove (b), it suffices to show that if $\operatorname{Ext}_{k Q}^{1}\left(\tau U_{1}, M\right) \neq 0$, then a basis vector $\xi$ of this vector space factors through $\tau T$.

We construct a morphism $f$ from $\tau T$ to $M$. Indeed, the sectional path in $\mathcal{T}$ from the mouth to $M$ and the sectional path from $\tau T_{1}$ to the mouth intersect in an indecomposable summand $\tau T_{i}$ of $\tau T$. Let $f$ denote the composition of the morphisms lying on the sectional path from $\tau T_{i}$ to $M$. Then $f \neq 0$ (see [5, Corollary IX 2.2]).

Now, observe that we have a non-split short exact sequence in $\bmod k Q$

$$
\zeta: 0 \longrightarrow \tau T_{i} \longrightarrow E \longrightarrow \tau U_{1} \longrightarrow 0
$$

with $E$ indecomposable. Because $\operatorname{Ext}_{k Q}^{1}\left(\tau U_{1}, M\right)$ is one dimensional, there exists a scalar $\lambda \in k$ such that $\xi=\zeta(\lambda f)$, that is, we have a commutative diagram with exact rows

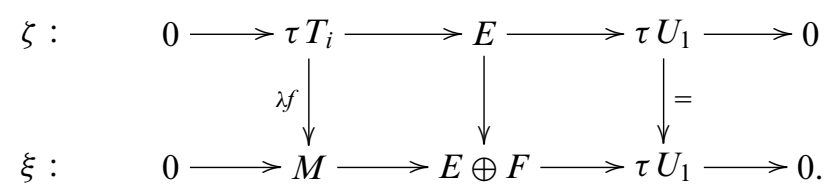


Indeed, the middle term of $\xi$ is of this form as seen in the picture below.

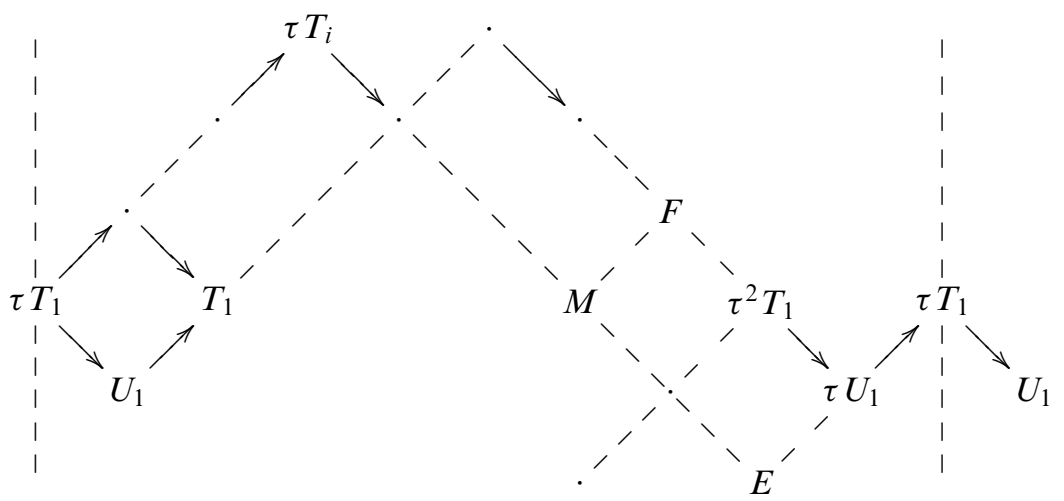

The fact that $\xi=\zeta(\lambda f)$ shows that $\xi$ factors through $\tau T$ in $\mathcal{C}$. This completes the proof.

COROLlary 4.4. With the above notation, letting $S_{1}=\operatorname{top} P_{1}$, we have $\left\langle S_{1}, N\right\rangle=1$ for all $N \in \Omega$.

Proof. Recall that $\left\langle S_{1}, N\right\rangle=\operatorname{dim} \operatorname{Hom}_{B}\left(S_{1}, N\right)-\operatorname{dim}_{\operatorname{Ext}_{B}^{1}}^{1}\left(S_{1}, N\right)$. Now, applying the functor $\operatorname{Hom}_{B}(-, N)$ to the short exact sequence

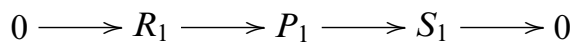

in $\bmod B$ yields an exact sequence

$$
0 \longrightarrow \operatorname{Hom}_{B}\left(S_{1}, N\right) \longrightarrow \operatorname{Hom}_{B}\left(P_{1}, N\right) \rightarrow \operatorname{Hom}_{B}\left(R_{1}, N\right) \rightarrow \operatorname{Ext}_{B}^{1}\left(S_{1}, N\right) \longrightarrow 0
$$

from which we deduce $\left\langle S_{1}, N\right\rangle=\operatorname{dim} \operatorname{Hom}_{B}\left(P_{1}, N\right)-\operatorname{dim} \operatorname{Hom}_{B}\left(R_{1}, N\right)$, and the latter equals 1 because of the lemma.

4.3. Proof of Theorem 1.6(b). We are now in a position to prove the second part of our Theorem 1.6.

THEOREM 4.5. Let $\mathcal{A}$ be a cluster algebra of Euclidean type and $x, x^{\prime}$ be two cluster variables with $x^{\prime}$ regular. Then $x$ and $x^{\prime}$ are compatible if and only if, for any cluster $\mathbf{x}$ containing $x$, the Laurent expansion $\mathcal{L}\left(x^{\prime}, \mathbf{x}\right)$ has no $x$ in its denominator.

Proof. Because of Lemma 3.1, we only need to prove the sufficiency. Assume that $x, x^{\prime}$ are not compatible, with $x^{\prime}$ regular. Because of Theorem 3.3, we may assume that $x$ is regular as well. Since there are no extensions between different tubes, we may assume that they correspond to objects in the cluster category lying in the same tube $\mathcal{T}$. We denote by $r$ the rank of $\mathcal{T}$. Let $T_{1}$ and $M$ be such that $X_{T_{1}[1]}^{T}=x$ and $X_{M}^{T}=x^{\prime}$. If the quasi-length of $T_{1}$ is at most $r-2$, then because of [8], we have $\operatorname{den} x^{\prime}=\operatorname{dim} \operatorname{Hom}_{\mathcal{C}}(T, M)$. Applying the same argument as in the proof of Theorem 3.3 yields the conclusion.

Thus, assume that the quasi-length of $T_{1}$ is $r-1$. We may assume that the objects lying on the sectional path from $T_{1}$ to the mouth are summands of the tilting 
object $T$. Lemma 3.2 implies that $\operatorname{Hom}_{\mathcal{C}}\left(T_{1}, M\right) \neq 0$, and since $M$ is an exceptional object in $\mathcal{T}$, we have $M \in \Delta$. We now recall that

$$
X_{M}^{T}=\sum_{\mathbf{e} \in \mathbb{N}^{\mathbb{Q}_{0}}} \chi\left(\operatorname{Gr}_{\mathbf{e}}\left(\operatorname{Hom}_{\mathcal{C}}(T, M)\right) \prod_{i \in Q_{0}} x_{i}^{\left\langle S_{i}, \mathbf{e}\right)_{a}-\left\langle S_{i}, \operatorname{Hom}_{\mathcal{C}}(T, M)\right\rangle} .\right.
$$

The summand corresponding to the vector $\mathbf{e}=0$ is

$$
\prod_{i \in Q_{0}} x_{i}^{-\left\langle S_{i}, \operatorname{Hom}_{\mathcal{C}}(T, M)\right\rangle}
$$

Because of Corollary 4.4, we have

$$
\left\langle S_{1}, \operatorname{Hom}_{\mathcal{C}}(T, M)\right\rangle=1 .
$$

In other words, the Laurent monomial

$$
\prod_{i \in Q_{0}} x_{i}^{-\left\langle S_{i}, \operatorname{Hom}_{\mathcal{C}}(T, M)\right\rangle}
$$

contains the variable $x\left(=x_{1}\right)$ in its denominator. Because of the positivity theorem (see [15]), this Laurent monomial is not cancelled by other summands. Therefore, we have

$$
X_{M}^{T}=\frac{p(\mathbf{x})}{x^{d} m(\mathbf{x} \backslash\{x\})}
$$

with $d>0$. This completes the proof.

\section{Unistructurality and automorphisms.}

5.1. We now define the notion of unistructurality.

Let $\mathcal{A}=\mathcal{A}(\mathbf{x}, Q)$ be a cluster algebra with initial seed $(\mathbf{x}, Q)$. This initial seed and the mutation process yield a family $\left(\mathbf{x}_{\alpha}\right)_{\alpha \in \Gamma_{0}}$ of clusters, where $\Gamma_{0}$ is the set of vertices of the corresponding exchange graph $\Gamma$. Let $\mathcal{X}=\cup_{\alpha \in \Gamma_{0}} \mathbf{x}_{\alpha}$ be the set of cluster variables.

We say that $\mathcal{A}$ is unistructural if, for any subset $\mathbf{x}^{\prime}$ of $\mathcal{X}$ and quiver $Q^{\prime}$ such that the pair $\left(\mathbf{x}^{\prime}, Q^{\prime}\right)$ generates by mutation the same set $\mathcal{X}$ of cluster variables, the exchange graphs and the set of clusters of $\mathcal{A}$ and $\mathcal{A}\left(\mathbf{x}^{\prime}, Q^{\prime}\right)$ are the same. More precisely, if $\left(\mathbf{x}^{\prime}, Q^{\prime}\right)$ generates by mutation a family of clusters $\left(\mathbf{x}_{\beta}^{\prime}\right)_{\beta \in \Gamma_{0}^{\prime}}$, where $\Gamma_{0}^{\prime}$ is the set of vertices of the corresponding exchange graph $\Gamma^{\prime}$, then the equality $\mathcal{X}=\cup_{\beta \in \Gamma_{0}^{\prime}} \mathbf{x}_{\beta}^{\prime}$ of the set of cluster variables implies that $\Gamma=\Gamma^{\prime}$ and there exists a permutation $\sigma$ of $\Gamma_{0}$ such that $\mathbf{x}_{\alpha}=\mathbf{x}_{\sigma(\alpha)}^{\prime}$ for any $\alpha$.

We also say that a cluster algebra $\mathcal{A}=\mathcal{A}(\mathbf{x}, Q)$ is weakly unistructural if its set of cluster variables cannot be obtained as a set generated by mutations starting with a seed $\left(\mathbf{x}^{\prime}, Q^{\prime}\right)$, where $Q^{\prime}$ is mutation equivalent to $Q$ but $\mathbf{x}^{\prime}$ is not a cluster of $\mathcal{A}$. In other words, the above definition of unistructural cluster algebra reduces to that of weakly unistructural if instead of allowing $Q^{\prime}$ to be an arbitrary quiver we restrict it to be a quiver mutation equivalent to $Q$.

Note that under the hypothesis that $\mathcal{X}=\cup_{\beta \in \Gamma_{0}^{\prime}} \mathbf{x}_{\beta}^{\prime}$, the ranks of $\mathcal{A}$ and $\mathcal{A}\left(\mathbf{x}^{\prime}, Q^{\prime}\right)$ are necessarily the same: Indeed, this rank is the cardinality of a transcendence basis of the (common) ambient field. 
As stated in the Introduction, we conjecture that every cluster algebra is unistructural. In this section, we prove that weak unistructurality can be expressed by means of cluster automorphisms. To this end, we start by recalling the following definition.

Definition 1. [4] Let $\mathcal{A}$ be a cluster algebra and $f: \mathcal{A} \rightarrow \mathcal{A}$ be an automorphism of $\mathbb{Z}$-algebras. Then $f$ is a cluster automorphism if there exists a seed $(\mathbf{x}, Q)$ in $\mathcal{A}$ such that the following conditions are satisfied.

(1) $f(\mathbf{x})$ is a cluster, and

(2) $f$ is compatible with mutations, that is, for every cluster variable $x \in \mathbf{x}$, we have $f \mu_{x}=\mu_{f(x)} f$.

We refer to [4] for further properties of cluster automorphisms. Note however, that a cluster automorphism can be alternatively defined as an automorphism of $\mathbb{Z}$-algebras which maps every cluster to a cluster. In particular, it maps cluster variables to cluster variables, thus permuting the set of all cluster variables. We are interested here in the converse implication, and we prove that it holds true if and only if the given cluster algebra is weakly unistructural (Theorem 1.4 from the Introduction).

Proof of Theorem 1.4. Let $\mathcal{A}=\mathcal{A}(\mathbf{x}, Q)$ be a cluster algebra for which Conjecture 1.1 holds. Suppose there exists a seed $(\mathbf{y}, Q)$ which generates by mutation an algebra $\mathcal{A}^{\prime}=\mathcal{A}(\mathbf{y}, Q)$ whose set of cluster variables coincides with that of $\mathcal{A}$. Suppose also that $\mathbf{y}$ is not a cluster of $\mathcal{A}$ (that is, suppose $\mathcal{A}$ is not weakly unistructural). Let $f: \mathcal{A} \rightarrow \mathcal{A}$ be the unique automorphism of $\mathbb{Z}$-algebras such that $f\left(x_{i}\right)=y_{i}$ with $x_{i} \in \mathbf{x}$ and $y_{i} \in \mathbf{y}$ are the cluster variables corresponding to the same point $i$ of $Q$.

Since the seeds $(\mathbf{x}, Q)$ and $(\mathbf{y}, Q)$ have the same quiver, and $f$ induces a homomorphism of the ambient field, the variables obtained by successive mutations of the seed $(\mathbf{y}, Q)$ are exactly the images under $f$ of the cluster variables obtained by the corresponding mutations of the seed $(\mathbf{x}, Q)$. Since the two seeds generate by mutation the same set of cluster variables, we conclude that $f$ maps the set of cluster variables of $\mathcal{A}$ (and of $\mathcal{A}^{\prime}$ ) to itself. By our hypothesis, this implies that $f$ is a cluster automorphism. Thus, it maps clusters to clusters and therefore $\mathbf{y}$ is a cluster of $\mathcal{A}$. Therefore, $\mathcal{A}=\mathcal{A}^{\prime}$ as cluster algebras.

Conversely, let $\mathcal{A}$ be a weakly unistructural cluster algebra, and let $f: \mathcal{A} \rightarrow \mathcal{A}$ be an automorphism of the ambient field permuting the set of cluster variables. Let $\mathbf{x}=\left(x_{1}, \ldots, x_{n}\right)$ and $(\mathbf{x}, Q)$ be a seed of the cluster algebra $\mathcal{A}$. Consider a cluster algebra $\mathcal{A}_{1}$ generated by the initial seed $(f(\mathbf{x}), Q)$, where the variable $f\left(x_{i}\right)$ is associated with the same point $i \in Q_{0}$ as variable $x_{i}$ in seed $(\mathbf{x}, Q)$.

Since the two seeds have the same quiver $Q$, and $f$ is a homomorphism of the field, the variables obtained by successive mutations of the seed $(f(\mathbf{x}), Q)$ are exactly the images under $f$ of the cluster variables obtained by the corresponding mutations of the seed $(\mathbf{x}, Q)$.

Therefore, since $f$ is an automorphism permuting the cluster variables of $\mathcal{A}$, the sets of cluster variables of $\mathcal{A}$ and $\mathcal{A}_{1}$ coincide and $\mathcal{A}=\mathcal{A}_{1}$ as $\mathbb{Z}$-algebras. Because $\mathcal{A}$ is weakly unistructural, its set of cluster variables cannot be obtained by mutation from the seed $(f(\mathbf{x}), Q)$ with $f(\mathbf{x})$ not being a cluster of $\mathcal{A}$. Thus, $f(\mathbf{x})$ is a cluster of $\mathcal{A}$. By Corollary 2.7 of [4], $f$ is a cluster automorphism.

LEMMA 5.1. Let $\mathcal{A}$ be a cluster algebra of Dynkin type, then $\mathcal{A}$ is unistructural if and only if $\mathcal{A}$ is weakly unistructural. 
Proof. Observe that the rank $n$ of $\mathcal{A}$ is determined by the set of cluster variables. This is the transcendence degree of the ambient field. The number of cluster variables is $n(n+3) / 2$ in the case $\mathbb{A}_{n}$, it is $n^{2}$ in the case $\mathbb{D}_{n}$ and is equal to 42,70 or 128 in the cases $\mathbb{E}_{6}, \mathbb{E}_{7}$ or $\mathbb{E}_{8}$ respectively. Therefore, the number of cluster variables determines the type.

Thus, if $\mathcal{A}_{1}$ is another cluster algebra which has the same set of cluster variables as $\mathcal{A}$, then $\mathcal{A}$ and $\mathcal{A}_{1}$ are of the same type. In particular, if $\mathcal{A}$ is weakly unistructural, then $\mathcal{A}$ is unistructural. The other implication is obvious.

COROLLARY 5.2. The statement of Conjecture 1.3 holds for cluster algebras of Dynkin type.

Proof. This follows directly from the lemma and Theorem 1.4.

We end the paper by proving that cluster algebras of rank 2 are unistructural.

THEOREM 5.3. Let $\mathcal{A}$ be a cluster algebra of rank 2. Then $\mathcal{A}$ is unistructural.

Proof. Let us fix a cluster structure on $\mathcal{A}$. Let $\left(\left(x_{1}, x_{2}\right), Q\right)$ be the initial seed, where $Q$ denotes the quiver $Q=1 \stackrel{r}{\rightarrow} 2$ with $r \geq 0$ the number of arrows from 1 to 2 .

Let first $r=0$. In this case the algebra $\mathcal{A}$ contains four clusters $\left(x_{1}, x_{2}\right),\left(\frac{2}{x_{1}}, x_{2}\right)$, $\left(\frac{2}{x_{1}}, \frac{2}{x_{2}}\right)$ and $\left(x_{1}, \frac{2}{x_{2}}\right)$. Suppose there is a second cluster structure with the same set of cluster variables and consider its cluster containing $x_{1}$. The only possible combination of two cluster variables of $\mathcal{A}$ which is not a cluster of $\mathcal{A}$ is $\left(x_{1}, \frac{2}{x_{1}}\right)$, but this set cannot be a cluster since it is not a transcendence basis of the ambient field of fractions in two variables $x_{1}, x_{2}$.

Let now $r \geq 1$. Using the expansion formula of [16], we see that the only cluster variables that do not have both $x_{1}$ and $x_{2}$ in the denominator are $x_{1}, x_{2}, \frac{x_{1}^{r}+1}{x_{2}}, \frac{x_{2}^{r}+1}{x_{1}}$. Moreover, there are exactly two clusters containing $x_{1}$, namely $\left(x_{1}, x_{2}\right)$ and $\left(x_{1}, \frac{x_{1}^{r}+1}{x_{2}}\right)$. Suppose there is another cluster structure of $\operatorname{rank} 2$ on $\mathcal{A}$ with the same set of variables. Then there exists a cluster $\left(x_{1}, y\right)$ with

$$
y=\mathcal{L}\left(y,\left(x_{1}, x_{2}\right)\right)=\frac{P\left(x_{1}, x_{2}\right)}{x_{1}^{d_{1}} x_{2}^{d_{2}}}
$$

such that $d_{1}>0$ and $P$ is a polynomial in $\mathbb{Z}\left[x_{1}, x_{2}\right]$, which is not divisible by $x_{1}$. Mutating this cluster in direction $y$ produces the following cluster

$$
\left(x_{1}, \frac{x_{1}^{m}+1}{P\left(x_{1}, x_{2}\right)} x_{1}^{d_{1}} x_{2}^{d_{2}}\right),
$$

where $m$ is the number of arrows in the quiver of the cluster $\left(x_{1}, y\right)$ in the second cluster structure. The Laurent phenomenon implies that

$$
P\left(x_{1}, x_{2}\right)=Q\left(x_{1}, x_{2}\right) M\left(x_{1}, x_{2}\right),
$$

where $M$ is a monomial and $Q$ is a divisor of $x_{1}^{m}+1$ in $\mathbb{Z}\left[x_{1}, x_{2}\right]$. Moreover, $x_{1}$ does not divide $M$ because it does not divide $P$. We consider two cases. If $M=1$ then $P=Q$ 
and so our cluster is

$$
\left(x_{1}, \frac{x_{1}^{m}+1}{P\left(x_{1}, x_{2}\right)} x_{1}^{d_{1}} x_{2}^{d_{2}}\right)=\left(x_{1}, P^{\prime}\left(x_{1}, x_{2}\right)\right),
$$

where $P^{\prime}$ is a polynomial in $\mathbb{Z}\left[x_{1}, x_{2}\right]$. Because of our description of cluster variables, it follows that $P^{\prime}=x_{2}$. If $M \neq 1$ then, because $x_{1}$ does not divide $M, M=x_{2}^{e_{2}}$ with $e_{2} \geq 1$. Thus, the denominator of the cluster variable $\frac{x_{1}^{m}+1}{P\left(x_{1}, x_{2}\right)} x_{1}^{d_{1}} x_{2}^{d_{2}}$ is equal to $x_{2}^{-d_{2}+e_{2}}$. Again, because of our description of cluster variables, this cluster variable is equal to $\frac{x_{1}^{r}+1}{x_{2}}$. This implies that $d_{1}=0$, a contradiction.

ACKNOWLEDGEMENTS. The first and third authors gratefully acknowledge partial support from NSERC of Canada, FRQNT and the University of Sherbrooke. The second author was supported by NSF grants 1001637 and 1254567, and by the University of Connecticut.

\section{REFERENCES}

1. I. Assem, T. Brüstle and R. Schiffler, Cluster-tilted algebras and slices, J. Algebra 319 (2008), 3464-3479.

2. I. Assem, T. Brüstle and R. Schiffler, Cluster-tilted algebras without clusters, J. Algebra 324, (2010) 2475-2502.

3. I. Assem and G. Dupont, Modules over cluster-tilted algebras determined by their dimension vectors. Comm. Algebra 41(2) (2013), 4711-4721.

4. I. Assem, R. Schiffler and V. Shramchenko, Cluster automorphisms, Proc. London Math. Soc. 3(104) (2012), 1271-1302.

5. I. Assem, D. Simson and A. Skowroński, Elements of the representation theory of associative algebras, 1: Techniques of representation theory, London Mathematical Society Student Texts, vol. 65 (Cambridge University Press, Cambridge, UK, 2006).

6. A. B. Buan and R. Marsh, Denominators in cluster algebras of affine type, J. Algebra, 323(8) (2010), 2083-2102.

7. A. B. Buan, R. Marsh, M. Reineke, I. Reiten and G. Todorov, Tilting theory and cluster combinatorics, Adv. Math. 204(2) (2006), 572-618.

8. A. B. Buan, R. Marsh and I. Reiten, Denominators of cluster variables, J. London Math. Soc. 79(3) (2009), 589-611. doi: 10.1112/jlms/jdn082.

9. A. B. Buan, R. Marsh and I. Reiten, Cluster-tilted algebras, Trans. Amer. Math. Soc. 359(1), (2007), 323-332.

10. P. Caldero and B. Keller. From triangulated categories to cluster algebras II. Ann. Sc. Ec. Norm. Sup. 39(4) (2006), 83-100.

11. S. Fomin, M. Shapiro and D. Thurston, Cluster algebras and triangulated surfaces. Part I: Cluster complexes, Acta Math. 201 (2008), 83-146.

12. S. Fomin and A. Zelevinsky, Cluster algebras I. Foundations, J. Amer. Math. Soc. 15(2) (2002), 497-529 (electronic).

13. S. Fomin and A. Zelevinsky, Cluster algebras IV: Coefficients, Compos. Math. 143 (2007), 112-164.

14. Y. Kimura and F. Qin, Graded quiver varieties, quantum cluster algebras, and dual canonical basis, arXiv:1205.2066v2.

15. K. Lee and R. Schiffler, Positivity for cluster algebras, arXiv:1306.2415.

16. K. Lee and R. Schiffler, A combinatorial algebra for rank 2 cluster variables, J. Algebra Comb. 37(1), (2013), 67-85.

17. G. Musiker, R. Schiffler and L. Williams, Positivity for cluster algebras from surfaces, Compos. Math. 149(2) (2013), 217-263.

18. Y. Palu, Cluster characters II: A multiplication formula, Proc. London Math. Soc. 104(1) (2012), 57-78.

19. I. Saleh, On the automorphisms of cluster algebras, arXiv: 1011.0894 . 


\section{Addendum: Note added in proof}

\section{ADDENDUM TO 'CLUSTER AUTOMORPHISMS AND COMPATIBILITY OF CLUSTER VARIABLES’}

\section{IBRAHIM ASSEM, RALF SCHIFFLER AND VASILISA SHRAMCHENKO}

We prove that Conjectures 1.1 and 1.2 hold true for cluster algebras of Dynkin type. For definitions and notations, we refer to [ASS2].

TheOREM 0.1. Let $\mathcal{A}$ be a cluster algebra of Dynkin type, then $\mathcal{A}$ is unistructural.

Proof. Assume that $\mathcal{A}$ is given two cluster structures $\mathcal{X}=\cup_{\alpha} \mathbf{x}_{\alpha}=\cup_{\beta} \mathbf{x}_{\beta}^{\prime}$, where $\mathbf{x}_{\alpha}$ and $\mathbf{x}_{\beta}^{\prime}$ are clusters in their respective structures. Denote the two cluster structures by $S$ and $S^{\prime}$, respectively. Let $\mathbf{x}=\left\{x_{1}, x_{2}, \ldots, x_{n}\right\}$ be the initial cluster of $S$. We claim that $\mathbf{x}$ is also a cluster in $S^{\prime}$. If not, then there exist two initial variables $x_{i}, x_{j}$ which are not compatible in $S^{\prime}$ : indeed, this can be seen by using the well-known bijections between clusters and tilting objects in the associated cluster category, and between cluster variables and rigid indecomposable objects. Because of the positivity theorem, each of $x_{i}$ and $x_{j}$ is a positive element in $\mathcal{A}$ (in both structures), hence so is their product $x_{i} x_{j}$. Cerulli-Irelli showed that the cluster monomials form an atomic basis for $\mathcal{A}$, see [C] Th.1.1, which implies that every positive element is a linear combination of cluster monomials with non-negative coefficients.

Therefore, the product $x_{i} x_{j}$ in the structure $S^{\prime}$ can be written as a positive linear combination of cluster monomials: $x_{i} x_{j}=\sum \lambda_{M^{\prime}} M^{\prime}$. Each of the cluster monomials $M^{\prime}$ is a product of $S^{\prime}$-compatible cluster variables and each of these cluster variables can be written as a positive Laurent polynomial in $x_{1}, \ldots, x_{n}$, because the latter is a cluster of $S$ and both structures have the same set of cluster variables. Thus, the cluster monomial $M^{\prime}$ can also be written as a positive Laurent polynomial $\mathcal{L}\left(M^{\prime}\right)$ in $\left\{x_{1}, \ldots, x_{n}\right\}$.

Replacing each $M^{\prime}$ by $\mathcal{L}\left(M^{\prime}\right)$ in the sum $\sum \lambda_{M^{\prime}} M^{\prime}$, we get

$$
x_{i} x_{j}=\sum \lambda_{M^{\prime}} \mathcal{L}\left(M^{\prime}\right)
$$

and because of positivity there is no cancellation of terms in the right-hand side. Therefore, the sum $\sum \lambda_{M^{\prime}} M^{\prime}$ has only one term $M^{\prime}=x_{i} x_{j}$ and $\lambda_{M^{\prime}}=1$. But this means that $x_{i}$ and $x_{j}$ are $S^{\prime}$-compatible, a contradiction. This proves that $\left\{x_{1}, \ldots, x_{n}\right\}$ is a cluster in the structure $S^{\prime}$.

In order to complete the proof, it suffices to show that the quiver $Q^{\prime}$ of the cluster $\left\{x_{1}, \ldots, x_{n}\right\}$ in the structure $S^{\prime}$ is equal or opposite to the quiver $Q$ of the same cluster in the structure $S$. The mutations $\mu_{i}$ and $\mu_{i}^{\prime}$ in the direction $i$ applied to the cluster $\left\{x_{1}, \ldots, x_{n}\right\}$ in both structures $S$ and $S^{\prime}$ will produce a variable whose denominator is $x_{i}$. Namely,

$$
\mu_{i}\left(x_{i}\right)=\frac{\prod_{i \rightarrow j \text { in } Q} x_{j}+\prod_{i \leftarrow j \text { in } Q} x_{j}}{x_{i}} \quad \text { and } \quad \mu_{i}^{\prime}\left(x_{i}\right)=\frac{\prod_{i \rightarrow j \text { in } Q^{\prime}} x_{j}+\prod_{i \leftarrow j \text { in } Q^{\prime}} x_{j}}{x_{i}} .
$$

Since both cluster structures have the same cluster variables and since in the Dynkin case the denominators determine the cluster variables, it follows that $\mu_{i}\left(x_{i}\right)=\mu_{i}^{\prime}\left(x_{i}\right)$ 
and therefore either

$$
\prod_{i \rightarrow j \text { in } Q} x_{j}=\prod_{i \rightarrow j \text { in } Q^{\prime}} x_{j} \quad \text { and } \quad \prod_{i \leftarrow j \text { in } Q} x_{j}=\prod_{i \leftarrow j \text { in } Q^{\prime}} x_{j}
$$

or

$$
\prod_{i \rightarrow j \text { in } Q} x_{j}=\prod_{i \leftarrow j \text { in } Q^{\prime}} x_{j} \quad \text { and } \quad \prod_{i \leftarrow j \text { in } Q} x_{j}=\prod_{i \rightarrow j \text { in } Q^{\prime}} x_{j} .
$$

Since $i$ is arbitrary and $Q$ is connected, this implies that $Q=Q^{\prime}$ or $Q=Q^{\prime o p}$.

COROLlary 0.2. Conjecture 1.1 holds true for cluster algebras of Dynkin type.

Proof. This follows from the previous theorem and Theorem 1.4.

\section{REFERENCES}

ASS2. I. Assem, R. Schiffler and V. Shramchenko, Cluster automorphisms and compatibility of cluster variables, Glasgow Math. J.

C. G. Cerulli Irelli, Positivity in skew-symmetric cluster algebras of finite type, arxiv: 1102.3050. 